nephron

Experimental

Nephrology

and Genetics
Nephron 2018;138:310-323

DOI: $10.1159 / 000484096$
Received: May 26, 2017

Accepted after revision: October 9, 2017

Published online: January 17, 2018

\title{
The von Hippel-Lindau Gene Is Required to Maintain Renal Proximal Tubule and Glomerulus Integrity in Zebrafish Larvae
}

\author{
Ellen van Rooijen ${ }^{a, b} \quad$ Glenn van de Hoek ${ }^{c, d}$ Ive Logister ${ }^{a, b}$ Henry Ajzenberg ${ }^{a, c}$ \\ Nine V.A.M. Knoers ${ }^{d}$ Freek van Eeden ${ }^{b}$ Emile E. Voest ${ }^{a}$ e \\ Stefan Schulte-Merker ${ }^{b, f, g}$ Rachel H. Giles ${ }^{a, c}$ \\ ${ }^{\text {a }}$ Department of Medical Oncology, University Medical Center Utrecht, Utrecht, The Netherlands; ${ }^{\mathrm{b}}$ Hubrecht \\ Institute, KNAW and UMC Utrecht, Utrecht, The Netherlands; ' ${ }^{\mathrm{C}}$ Department Nephrology and Hypertension, \\ Regenerative Medicine Center Utrecht, University Medical Center Utrecht, Utrecht, The Netherlands; ${ }^{\mathrm{d}}$ Department \\ of Genetics, University Medical Center Utrecht, Utrecht, The Netherlands; ${ }^{e}$ Medical Oncology, Netherlands Cancer \\ Institute, Amsterdam, The Netherlands; ${ }^{f}$ Institute of Cardiovascular Organogenesis and Regeneration, Faculty of \\ Medicine, WWU, Münster, Germany; ${ }^{9}$ CiM Cluster of Excellence (EXC1003-CiM), Münster, Germany
}

\section{Keywords \\ von Hippel-Lindau · Zebrafish · Kidney integrity · \\ Pronephros · Vesicle trafficking · Hypoxia}

\begin{abstract}
Background: von Hippel-Lindau (VHL) disease is characterized by the development of benign and malignant tumours in many organ systems, including renal cysts and clear cell renal cell carcinoma. It is not completely understood what underlies the development of renal pathology, and the use of murine $\mathrm{Vh} /$ models has been challenging due to limitations in disease conservation. We previously described a zebrafish model bearing inactivating mutations in the orthologue of the human VHL gene. Methods: We used histopathological and functional assays to investigate the pronephric and glomerular developmental defects in vh/mutant zebrafish, supported by human cell culture assays. Results: Here, we report that $v h /$ is required to maintain pronephric tubule and glomerulus integrity in zebrafish embryos. vhl mutant glomeruli are enlarged, cxcr4a+ capillary loops are dilated and the Bowman space is widened. While we did not observe
\end{abstract}

\begin{tabular}{ll}
\hline KARGER & $\begin{array}{l}\text { (c) } 2018 \text { The Author(s) } \\
\text { Published by S. Karger AG, Basel }\end{array}$ \\
E-Mail karger@karger.com & $\begin{array}{l}\text { This article is licensed under the Creative Commons Attri- } \\
\text { bution-NonCommercial-NoDerivatives 4.0 International } \\
\text { www.karger.com/nef }\end{array}$ \\
& $\begin{array}{l}\text { License (CC BY-NC-ND) (http://www.karger.com/Ser- } \\
\text { vices/OpenAccessLicense). Usage and distribution for }\end{array}$
\end{tabular}

pronephric cysts, the cells of the proximal convoluted and anterior proximal straight tubule are enlarged, periodic acid schiff (PAS) and Oil Red O positive, and display a clear cytoplasm after hematoxylin and eosine staining. Ultrastructural analysis showed the $\mathrm{vhl}^{-1-}$ tubule to accumulate large numbers of vesicles of variable size and electron density. Microinjection of the endocytic fluorescent marker AM1-43 in zebrafish embryos revealed an accumulation of endocytic vesicles in the $v h l$ mutant pronephric tubule, which we can recapitulate in human cells lacking VHL. Conclusions: Our data indicates that $\mathrm{vh} /$ is required to maintain pronephric tubule and glomerulus integrity during zebrafish development, and suggests a role for VHL in endocytic vesicle trafficking.

(c) 2018 The Author(s)

Published by S. Karger AG, Basel

\section{Introduction}

The von Hippel-Lindau (VHL) disease is characterized by the heterozygous inactivation of a single VHL allele, which predisposes to benign and malignant tumour de- 
velopment in many organ systems [1]. In the kidney, biallelic VHL inactivation results in the formation of numerous benign cysts in $\sim 75 \%$ of the patient population [2]. It is thought that clear cell renal cell carcinoma (ccRCC, $35-75 \%$ prevalence) develops from cells lining these premalignant renal tubular cysts; however, not all cysts develop ccRCC, and not all cases of ccRCC are preceded by cysts [3-5]. Therefore, the exact mechanisms behind the development of these disease aspects are currently debated. Several lines of evidence suggest multiple steps to be involved, including microtubule instability [6], loss of cilia (associated with cyst formation) [7-10], changes in the extracellular matrix [11-13], and constitutive activation of the hypoxia inducible transcription factor HIF [14].

Modeling renal aspects of VHL disease in rodents has been challenging; renal pathology was not observed in mice in which $V h l$ was inactivated in a systemic mosaic pattern [15], or in systemic $\mathrm{Vhl}^{+/-}$mice [16], which were also not more susceptible to streptozotocin-induced renal carcinogenesis [17]. Conditional inactivation of $V h l$ in the renal tubule failed to induce ccRCC in mice $[18,19]$; yet it led to some important insights into the development of renal cysts. Deletion of $V h l$ in the renal proximal tubule using a Cre recombinase under the control of a phosphoenolpyruvate carboxykinase (PEPCK) promoter resulted in a low incidence (around 30\% of mice over 12 months) of glomerular and tubular cysts in a HIF-1 1 independent, HIF-1 $\beta$ dependent manner [18]. Cre/lox site-specific recombination using the Ksp1.3 promotor (Cdh16) resulted in the conditional inactivation of $V h l$ throughout the renal epithelium (although rarely in the proximal tubules); yet renal neoplasms were not observed [19]. It was therefore postulated that additional VHL-independent events may be required that lead to the activation of other cancer signalling pathways. Accordingly, several groups have recently demonstrated the development of ccRCC in mice models upon the combined loss of $V h l$ and different genes; Harlander et al. [20] showed that combined deletion of $V h l, p 53$ and $R b 1$ specifically in renal epithelial cells in mice caused ccRCC arising from proximal tubule epithelial cells. Nargund et al. [21] demonstrated that activation of mTORC1 constituted a third driver event after loss of $\mathrm{Vhl}$ and Pbmrl in their ccRCC tumour model, and Bailey et al. [22] showed that Myc activation when combined with $V h l$ and $C d k n 2 a$ (Ink4a/Arf) deletion, produced murine kidney tumours that approximate human ccRCC. pVHL might also exert a broader role in maintaining renal integrity. Ding et al. [23] showed that selective deletion of $\mathrm{Vhl}$ in glomerular podocytes results in a CXCR4-dependent development of necrotizing glomerular vasculitis with prominent segmental fibrin deposits, also termed rapid degenerative glomerulonephritis in mice from 4 weeks of age. In primary renal proximal epithelial cells and mouse embryonic fibroblast, it was shown that only the combined inactivation of pVHL and glycogen synthase kinase beta resulted in the loss of pre-established primary cilia, likely through the activation of protein kinase Akt [24]. This was supported by in vivo data, where combined deletion of $V h l$ and the tumour suppressor Pten (a negative regulator of the phosphatidylinositol-3-kinase signalling pathway and thus Akt activity) in Ksp1.3-cre mice resulted in the robust formation of proliferative cysts with reduced cilia numbers [19]. In support, a triple knock-out conditional murine model inactivating $V h l, p 53$ and ciliary Kif3 $a$ resulted in neoplastic renal lesions [25].

Due to its anatomical simplicity of consisting of just 2 nephrons, the zebrafish embryonic kidney - or pronephros - has proven itself to be a valuable and relevant model for studies of kidney development and disease (reviewed by Drummond [26]; Wingert and Davidson [27]; van de Hoek et al. [28]). We have previously shown that zebrafish mutant in the VHL tumour suppressor develop key aspects of the human disease condition, including the activation of the HIF signalling pathway, the development of polycythemia and excessive neovascularization of the retina and brain, and recently, the loss of $v h l$ was also shown to recapitulate ccRCC characteristics in zebrafish [29]. Here we report that $v h l$ is required to maintain pronephric tubule and glomerulus integrity in zebrafish embryos. vhl mutant glomeruli are enlarged, capillary loops are dilated and the Bowman space is widened. While we did not observe pronephric cysts, the cells of the proximal convoluted and anterior proximal straight tubule (PST) are enlarged, PAS and Oil Red O positive, and display a clear cytoplasm after hematoxylin and eosine (H\&E) staining. Ultrastructural analysis revealed the $\mathrm{vhl}^{-/-}$tubule to accumulate large numbers of vesicles of unknown content. Confocal fluorescent imaging in zebrafish embryos and human VHL-deficient cell suggests a role for VHL in endocytic trafficking. Our results indicate that zebrafish $v h l$ mutants will contribute to the understanding of the complex nature of $V H L$-associated renal processes.

\section{Material and Methods}

\section{Zebrafish Lines}

The $v h l^{h u 2117}(\mathrm{Q} 23 \mathrm{X})$ and $v h l^{h u 2081}(\mathrm{C} 31 \mathrm{X})$ mutant alleles were isolated from the Hubrecht target-selected ENU-mutagenized F1 zebrafish library [30] and out-crossed into the TG $(k d r \text {-like:egfp })^{\mathrm{s} 843}$ 
[31] transgenic line. Transheterozygote embryos ( $v h l^{h u 2117}$ / $\left.v h l^{h u 2081}\right)$ and homozygous embryos ( $v h l^{h u 2117} / v h l^{h u 2117}$; $v h l^{h u 2081} /$ $v h l^{h u 2081}$ ) were used interchangeably in experimental assays, hereafter termed $v \mathrm{hl}^{-/-}$. Where indicated, embryos were anesthetized with MS222 (final concentration of $0.17 \mathrm{mg} / \mathrm{mL}$ ).

\section{In situ Hybridization}

Whole-mount in situ hybridizations were performed as described [32] with minor modifications. Antisense digoxygenin (Roche) labelled mRNA probe for $c x c r 4 a$ was transcribed as previously indicated [33]. Probes were purified with NucleoSpin RNA clean-up columns (Machery-Nagel). To improve probe penetration, larvae older than $5 \mathrm{dpf}$ were partially cut open at the level of the yolk sack extension after ProtK permeabilization. After in situ hybridization, pigmented embryos were incubated with $0.1 \mathrm{M}$ $\mathrm{K}_{2} \mathrm{Cr}_{2} \mathrm{O}_{7}$ in $5 \%$ acetic acid for 30 min, washed extensively with PBS- $0.1 \%$ Tween-20 (PBT) and subsequently bleached in a $1-3 \%$ $\mathrm{H}_{2} \mathrm{O}_{2}$-PBT solution in bright light for approximately $2 \mathrm{~h}$.

\section{VHLp30 mRNA Rescue}

Rescue experiments were performed as previously described [34]. Human VHLp30 mRNA was generated using the SP6 mMESSAGE mMACHINE kit (Ambion). VHLp30 mRNA, measuring 10 pg, was injected into the yolk of one-cell stage embryos. Following phenotypic analysis, embryos were genotyped by sequencing.

\section{BrdU Proliferation Assay and Immunohistochemistry}

Embryos were pulsed with $3 \mathrm{~mm}$ bromodeoxyuridine (BrdU; Sigma Aldrich) in embryo medium for $6 \mathrm{~h}$ at $28^{\circ} \mathrm{C}$. Embryos were fixed in $4 \%$ paraformaldehyde and BrdU-incorporation was detected with primary anti-BrdU antibody (1:100, DAKO) and secondary anti-mouse IgG HRP (1:300, DAKO) according to standard protocols [35].

\section{Pronephric Fluorescent Dye Uptake and Confocal Analysis}

Anesthetized embryos were embedded in $0.5 \%$ agarose and administered one nanoliter of a $35 \mathrm{mg} / \mathrm{mL}$ lysine-fixable tetramethylrhodamine conjugated 70k MW dextran (TAMRA, Molecular probes) solution by cardiac puncture at $7 \mathrm{dpf}$. Only embryos exhibiting TAMRA throughout the cardiovascular system immediately after injection were further analyzed. To allow sufficient time for pronephric clearance and tubular reabsorption of TAMRA, embryos were incubated for 5-7 $\mathrm{h}$ in embryo medium [36] at $28^{\circ} \mathrm{C}$. For confocal analysis, embryos were anesthetized and embedded in $0.5 \%$ agarose on a coverslip. Images were collected using

Fig. 1. vhl mutants display proximal tubule abnormalities. a Schematic representation of the zebrafish pronephros at 6-7 dpf after the segmentation model of Wingert et al. [39] and a' bright field image of a $7.5 \mathrm{dpf} v \mathrm{hl}^{-/-}$mutant zebrafish. b Bright field lateral view of the PT at $7.5 \mathrm{dpf}$. Compared to the smooth cuboidal lining of the PCT and PST in wild-type sibling, vhl mutant tubular cells are irregular with a grape-like or alveolar appearance, which is most pronounced in the PCT and anterior PST. c H\&E staining on paraffin cross sections reveal the enlarged $v h l^{-/-} \mathrm{PT}$ cells displaying a clearer cytoplasm compared to siblings at $7.5 \mathrm{dpf}$. Original magnification 20x. d Sagittal section of the PT at $7.5 \mathrm{dpf}$ shows the lining of the PCT and PST is composed of a single layer of smooth cuboi- either a Zeiss LSM510 or Leica DM IRE2 confocal microscope using a 20x oil objective at the same laser intensity.

\section{VEGF Receptor Tyrosine Kinase Inhibitor Treatment}

Embryos were treated with $10 \mu \mathrm{M}(12.5 \mathrm{mM}$ stock in DMSO) VEGF receptor tyrosine kinase inhibitor 676475 (Calbiochem) in embryo medium [36] at $28^{\circ} \mathrm{C}$ in 6 -well culture plates, containing $15 \mathrm{vhl}$ mutants and 15 siblings per well. Control embryos were incubated with the equivalent amount of DMSO solution under the same conditions. Experiments were performed in triplicate.

\section{AM1-43 Injection}

Siblings and $v h l$ mutants were sorted by phenotype at $5 \mathrm{dpf}$. Cardiac administration of $1 \mathrm{nl}$ of $5 \mu \mathrm{M} \mathrm{AM} 1-43$ was performed in 15 embryos of each phenotypic group. Embryos recovered for 24 hours prior to confocal imaging (as above). Genotype was confirmed by Sanger sequencing after termination of the experiment. Of some embryos, vibratome sections (Leica VT1000S) were made and counterstained with DAPI for improved imaging of the pronephros.

\section{LiCl Treatment}

Three-day-old $v h l$ mutants and siblings were incubated with $0.5,1,1.5,5$ and $10 \mathrm{~mm} \mathrm{LiCl}$ or $\mathrm{CaCl}_{2}$ (control) in embryo medium at $28^{\circ} \mathrm{C}$ in 6 -well culture plates, containing 30 embryos per well. Embryos were kept at $28^{\circ} \mathrm{C}$ and monitored daily for the development of pronephric cysts.

\section{Transmission Electron Microscopy}

Embryos were fixed in Karnovsky fixative (2\% paraformaldehyde, $2.5 \%$ glutaraldehyde, $0.08 \mathrm{M} \mathrm{Na}$-cacodylate $\mathrm{pH} 7.4,0.25 \mathrm{mM}$ calcium chloride, $0.5 \mathrm{mM}$ magnesium chloride set to $\mathrm{pH}$ 7.4) for at least $24 \mathrm{~h}$ at $4{ }^{\circ} \mathrm{C}$. Samples were postfixed in $1 \%$ osmiumtetroxide and embedded in Epon 812. Ultrathin sections $(60 \mathrm{~nm})$ were contrasted with $3 \%$ uranyl magnesium acetate and lead citrate and viewed with a Jeol JEM 1010 transmission electron microscope.

\section{Histology}

Plastic or paraffin sections $(7 \mu \mathrm{m})$ were stained with PAS, haematoxylin and/or eosin using standard protocols. Cryo-sections $(10 \mu \mathrm{m})$ were used for Oil Red $\mathrm{O}$ staining according to standard protocol. Blood cells were visualized by $o$-dianisidine staining of hemoglobin as described [37].

dal cells with apical brush border PAS staining in siblings, while $\mathrm{vhl}^{-/-}$affected tubular cells are completely PAS positive. Original magnification $20 \times$. e Occasionally, $v h l^{-/-}$PAS-positive cells (arrows) are observed in more distal pronephric segments. Original magnification $20 \times$. $\mathbf{f}$ Cross section of haematoxylin and Oil-Red-O staining shows increased lipid contents in the $v h l^{-1-}$ PT. Anterior is to the left in all images. PCT, proximal convoluted tubule; PST, proximal straight tubule; PT, proximal tubule (PCT + PST); DE, distal early; CS, corpuscle of Stannius; DL, distal late; PD, pronephric duct; C, cloaca; SB, swim bladder; PAS, periodic acid schiff; $\mathrm{H} \& \mathrm{E}$, haematoxylin and eosin; dpf, days post-fertilization.

(For figure see next page.) 


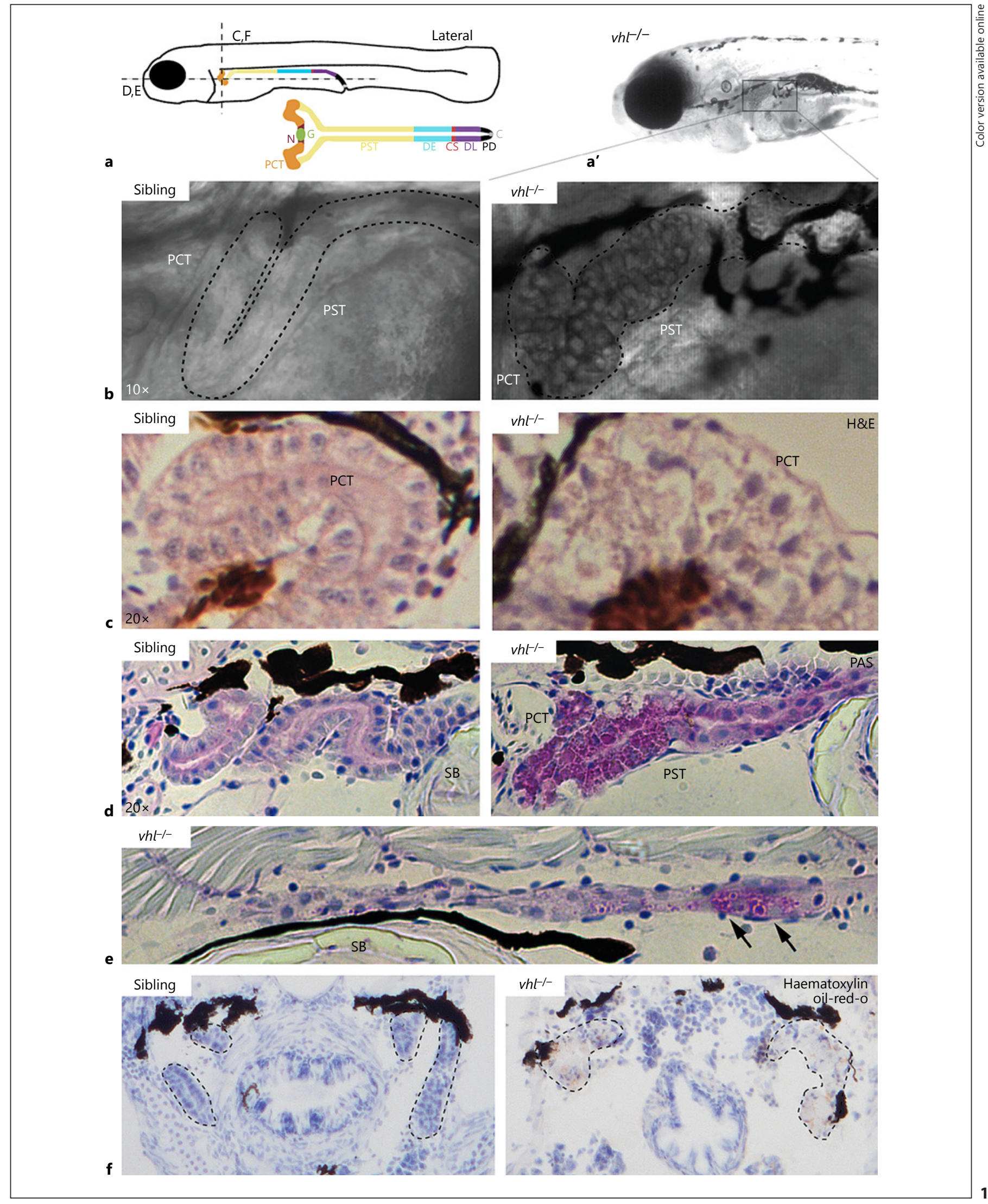


RAB7A Microinjections

786-0 cells were purchased from ATCC (CRL-1932) and cultured as suggested. Stable cell lines re-expressing the p30 isoform of VHL have been previously characterized [38]. The mCherryRAB7A construct was a kind gift from Chris Westlake. Microinjections using the FemtoJet (Eppendorf) and micropipette puller (P97, Sutter Instrument), and imaging 10 cells per condition was performed approximately $24 \mathrm{~h}$ later. Z-stacks were taken and the maximum diameter of vesicles from 2 independent experiments ( $n=30$ per experiment) was measured using the ruler tool on Zen software on a Zeiss LSM700 confocal microscope.

Ingenuity Pathway Analysis

DRSC Integrative Ortholog Prediction Tool (DIOPT) [58] was used on our zebrafish $v h l$ microarray gene ID list (previously published in van Rooijen et al. [34]) to identify human orthologs. The corresponding functional annotations of differentially expressed genes were interrogated using Ingenuity Pathway Analysis software (QIAGEN Inc., https://www.qiagenbioinformatics.com/ products/ingenuitypathway-analysis).

\section{Results}

vhl Mutants Develop Severe Proximal Tubule and Glomerular Abnormalities

The segmental organization of the zebrafish pronephros is very similar to the mammalian metanephros [39] (Fig. 1a). In live embryos, the smooth lining of the proximal convoluted (PCT) and straight (PST) tubule is easily visible from a lateral view (Fig. 1a, b). Strikingly, between 3 and 4 days post-fertilization (dpf), vhl mutant tubular cells develop an irregular and grape-like or alveolar appearance (online suppl. Fig. 1; for all online suppl. material, see www.karger.com/doi/10.1159/000484096), which is most pronounced in the neck (not shown), PCT and anterior PST, as shown in Figure 1b, c at 7.5 dpf. This phenotype can be rescued by injection with $10 \mathrm{pg}$ human VHLp30 mRNA (online suppl. Fig. 1).

Human $\mathrm{VHL}^{-/-}$ccRCC cells accumulate glycogen and as a consequence their cytoplasm appears clear when stained for $\mathrm{H} \& \mathrm{E}$ but stains positive for periodic acid schiff (PAS) [40]. To investigate whether $v h l$ mutant cells would share these distinct ccRCC characteristics, we performed a histopathological analysis of the affected proximal tubule. H\&E staining on paraffin-embedded sections revealed the large $v \mathrm{hl}^{-/-}$PCT cells to have a clearer cytoplasmic appearance when compared to a sibling cross-section at $7.5 \mathrm{dpf}$ (Fig. 1c). In siblings, the lining of the PCT and PST is composed of a single layer of smooth cuboidal cells with a PAS-positive apical brush border (Fig. 1d). Proximal tubule $\mathrm{vhl}^{-/-}$cells, however, are uniformly PAS-positive (Fig. 1d). This is most pronounced in the PCT and anterior PST, while more posterior staining is reduced and cells appear morphologically less affected (Fig. 1d). Interestingly, occasionally pink cells were observed among PAS-negative cells in the more distal pronephric segments of $v h l$ mutants (arrows, Fig. 1e), suggesting that other segments might be affected as well. Diastase treatment, however, did not alter $v \mathrm{hl}^{-/-}$PAS staining of the proximal tubule, demonstrating that the PAS staining in $v h l$ mutants is not due to glycogen depositions (not shown). Oil Red O staining suggest an increased lipid content in the enlarged PT cells (Fig. 1f). Additionally, Ingenuity Pathway Analysis (IPA) of $v \mathrm{hl}^{-/-}$versus wt sibling embryos (online suppl. Fig. 3) shows robust changes in molecular and cellular functions specifically pertaining to upregulated metabolism, synthesis and transport of lipids.

Histological examination furthermore revealed severe glomerular abnormalities in the vhl mutant (Fig. 2a-e). We observe that compared with glomeruli of age-matched siblings, the $v \mathrm{hl}^{-/-}$glomerulus is enlarged, the Bowman space is widened (double arrow) and the capillary loops are dilated (red arrowheads; Fig. $2 \mathrm{~b}$ ). $o$-Dianisidine staining revealed the capillary loops to contain blood cells and increased perfusion of the glomerulus to be present in $v h l$ mutants (Fig. 2c).

Deletion of $V h l$ in glomerular podocytes induced the formation of dilated capillary loops and de novo expression of $C x c r 4$ in mouse podocytes [23]. In zebrafish, Cxcr 4 is duplicated in $c x c r 4 a$ and $c x c r 4 b$, with both fulfilling distinct functions [33]. We have previously shown that $v h l$ mutants express cxcr $4 a$ in angiogenic blood vessels in the brain and retina, both tissues which, like the $v \mathrm{hl}^{-/-}$glomerulus, express high levels of vegf [41]. In Figure $2 \mathrm{~d}, c x$ cr4a-positive capillary loops can be observed. Furthermore, some smaller cells appear to express cxcr4a (arrows); however, it needs to be further investigated whether these represent podocytes. While a widened Bowman space was observed in $8 / 8$ histologically examined $v h l$ mutants, this is not observed in Figure 2d, due to in situ hybridization processing.

To investigate proliferation levels in the pronephros, we pulsed the embryos with BrdU. vhl mutants did not display an altered proliferation rate in the glomerulus and PT compared to siblings at $7.5 \mathrm{dpf}$, whereas brown proliferating cells could be readily observed in the $\mathrm{vhl}^{-/-}$pronephric hematopoietic tissue (Fig. 2e, red arrowheads).

\section{Accumulation of Vesicles in vhl -1- $^{-}$Proximal Tubule}

To investigate the cytoplasmic content and ultrastructural characteristics of the PT cells, we performed electron 


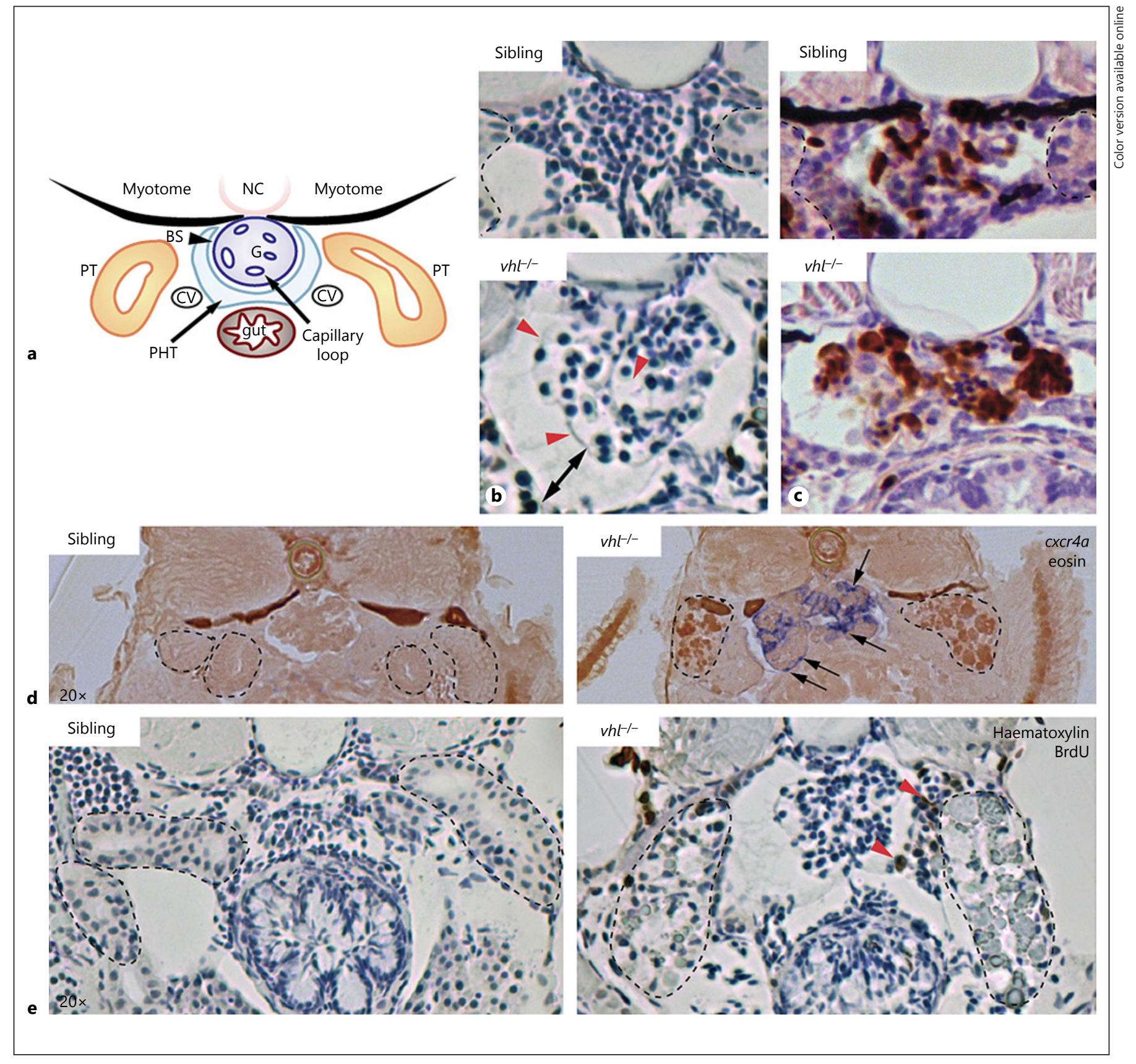

Fig. 2. vhl mutants display glomerular abnormalities. a Schematic representation of a cross section through a $7.5 \mathrm{dpf}$ zebrafish larva at the level of the glomerulus. b Compared to age-matched siblings, the $v \mathrm{hl}^{-/-}$Bowman space is widened (double arrow), the glomerulus is enlarged and dilated capillary loops (red arrowheads) are observed. c $o$-Dianisidine staining reveals capillary loops to contain blood cells and perfusion of the glomerulus to be increased in $v h l$ mutants. d In situ hybridization shows $c x c r 4 a$ to be expressed in the capillary loops and podocytes (arrows) of $v \mathrm{hl}$ mutants, while in siblings cxcr4a mRNA levels were too low to detect. e BrdU incorporation assays did not reveal altered proliferation of the affected $v h l^{-/}$glomerulus and PT compared to siblings. Increased proliferation is observed in the $v h l$ mutant PHT (red arrowheads). In all figures, dorsal is up and the proximal tubule is outlined for clarification. G, glomerulus; BS, Bowman space; PT, proximal tubule; $\mathrm{PHT}$, pronephric hematopoietic tissue; NC, notochord; CV, caudal vein. 


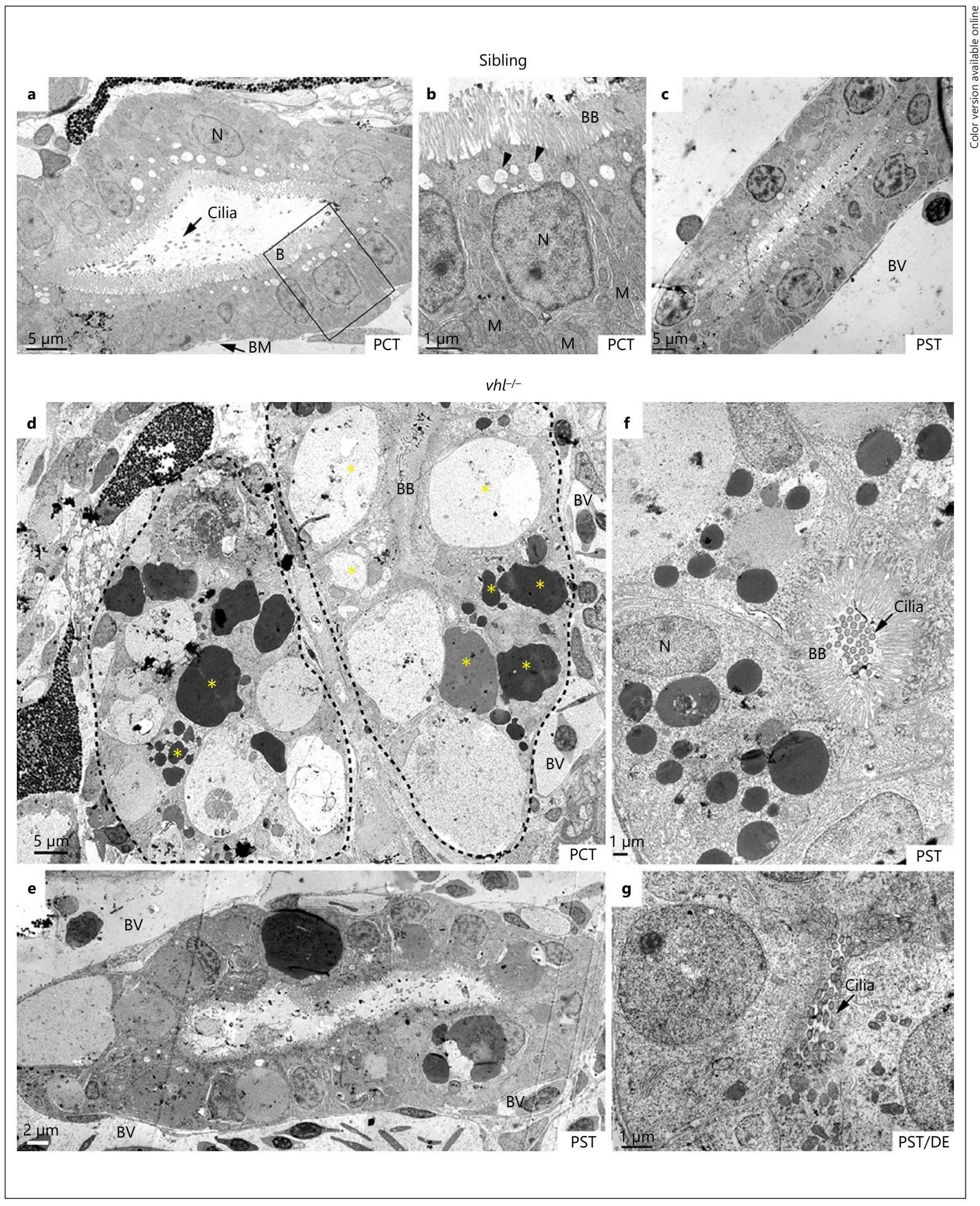

(For legend see next page.) 
microscopy analysis on $2 \mathrm{vhl}$ mutants and siblings at 7.5 dpf. In siblings, a regular organization of the polarized PCT cells is observed (Fig. 3a, b). At the apical side, brush border microvilli and cilia (arrow) reach into the tubular lumen, and endocytic vesicles (arrowheads) are observed close to the apical membrane (Fig. 3b). The nucleus and numerous mitochondria are located towards the basement membrane (Fig. 3b). The posterior PST showed a similar polarization; however, endocytic vesicles were less frequently observed (Fig. 3c). In $v h l$ mutants, PCT cells contain a striking quantity of vesicles (asterisks) of unknown content, variable in size and electron density, which are located throughout the cytoplasm (Fig. 3d). Cell boundaries or other organelles could hardly be discerned. Moving in a more distal direction from the PCT (from Fig. 3e-g) abnormalities gradually become less severe and at the level of the PST/DE no vesicles were observed (Fig. 3g). Electron micrographs of the pancreas, showed a similar accumulation of vesicles in the exocrine (zymogens) and endocrine (beta cells) tissues (online suppl. Fig. 4).

\section{Glomerular Filtration in $\mathrm{vhl}^{-/-}$Cells Is Not Impaired}

The injection of fluorescent compounds into the circulation is a well-established tool to visualize filtration by the zebrafish pronephros. It has been shown that biologically inert rhodamine-dextran conjugates are cleared by the glomerulus and subsequently reabsorbed by the proximal tubule by endocytosis (detectable in apical endosomes) or excreted via the cloaca [42-44]. To investigate whether the severely affected $v \mathrm{hl}^{-/-}$glomerulus and proximal tubules are able to exert these functions, we injected $7 \mathrm{dpf} T g$ ( $k d r$-like:egfp) vhl mutants and siblings with tetramethylrhodamine conjugated $70 \mathrm{k}$ MW dextran (TAMRA). Between 5 and $7 \mathrm{~h}$ post administration, embryos were analyzed. Confocal imaging of the proximal tubule revealed that in both mutants and siblings, TAMRA was cleared by the glomerulus (not shown) and taken up by the PT (Fig. 4a) or excreted via the cloaca (not shown). While in siblings TAMRA-containing vesicles

Fig. 3. Accumulation of vesicles in the $v h l^{-/-}$proximal tubule. Ultrastructural analysis of the proximal tubule at $7.5 \mathrm{dpf}$. a, b In siblings, a regular organization of the polarized PCT cells is observed. At the apical side, brush border microvilli and cilia (arrow) reach into the tubular lumen, and endocytic vesicles (arrowheads) are observed close to the apical membrane. Moving towards the basement membrane, the nucleus and numerous mitochondria are observed. c The posterior PST shows a similar polarization; however, endocytic vesicles are less frequently observed. d In vhl mutants, PCT cells contain a striking amount of vesicles (yellow asterisks) are small and appear to have a more apical distribution, vesicles in the $v \mathrm{hl}^{-/-} \mathrm{PT}$ are larger and fill up most of the PT cell lumen (left panels).

Neovascularization of the $\mathrm{vhl}^{-/-}$Proximal Tubule Does Not Contribute to the Aberrant Cell Morphology

Confocal analysis of the blood vasculature of the pronephric bed (Fig. 4a, middle and right panels) revealed severe neovascularization of the $v \mathrm{hl}^{-/-} \mathrm{PT}$. While in siblings the PT is wrapped around one main blood vessel, in vhl mutants, the PT is surrounded by blood vessels that form a fine cocoon-like structure over the tubular epithelium. To assess whether this network of blood vessels surrounding the $\mathrm{PT}$ and the general excess of vegf signalling might contribute to the aberrant tubular morphology, we treated embryos with the 676475 VEGFR-2 inhibitor (Calbiochem) that we previously showed to effectively block the enhanced angiogenic response in $v h l$ mutants [41]. Similarly, Figure $4 \mathrm{~b}$ shows that VEGF receptor inhibition from 2.5 to $5.75 \mathrm{dpf}$ blocked the $\mathrm{vhl}^{-/-}$PT neovascularization when compared to DMSO-treated mutants. Importantly, PT cell morphology was not obviously affected, indicating that the aberrant neovascularization or vegf overexpression do not contribute to this specific phenotype.

\section{vhl Mutants Show Endocytic Changes and Vesicle Trafficking}

To address the hypothesis that endocytosis may be affected in the $\mathrm{vhl}^{-/-}$pronephros, we performed cardiac administration of the styryl dye molecule AM1-43. AM1-43 is a fixable fluorescent activity-dependent endocytosis marker with a lipophilic tail and a hydrophilic, cationic head group, which is virtually nonfluorescent in aqueous solution [45]. When taken up by endocytosis, AM1-43 is strongly fluorescent. Upon cardiac injection of AM1-43 in $\mathrm{vhl}^{-1-}$ embryos, we observe a specific increase in dye uptake in the pronephric tubule as well as the intestine (Fig. 5a, b). In siblings, only mod-

of unknown content, variable in size and electron density, which are located throughout the cytoplasm. The PCT is outlined since individual cells could not be discerned. Moving in a more distal direction from the PCT (e-g), abnormalities gradually become less severe and brush border microvilli $(\mathbf{e}, \mathbf{f})$ and cilia of normal $9+2$ architecture (f) are observed. At the level of the posterior PST/DE (g), no abnormal vesicles are present. PCT, proximal convoluted tubule; PST, proximal straight tubule; PT, proximal tubule; DE, distal early; BB, brush border; $N$, nucleus; $M$, mitochondrion; BM, basement membrane; BV, blood vessel. 


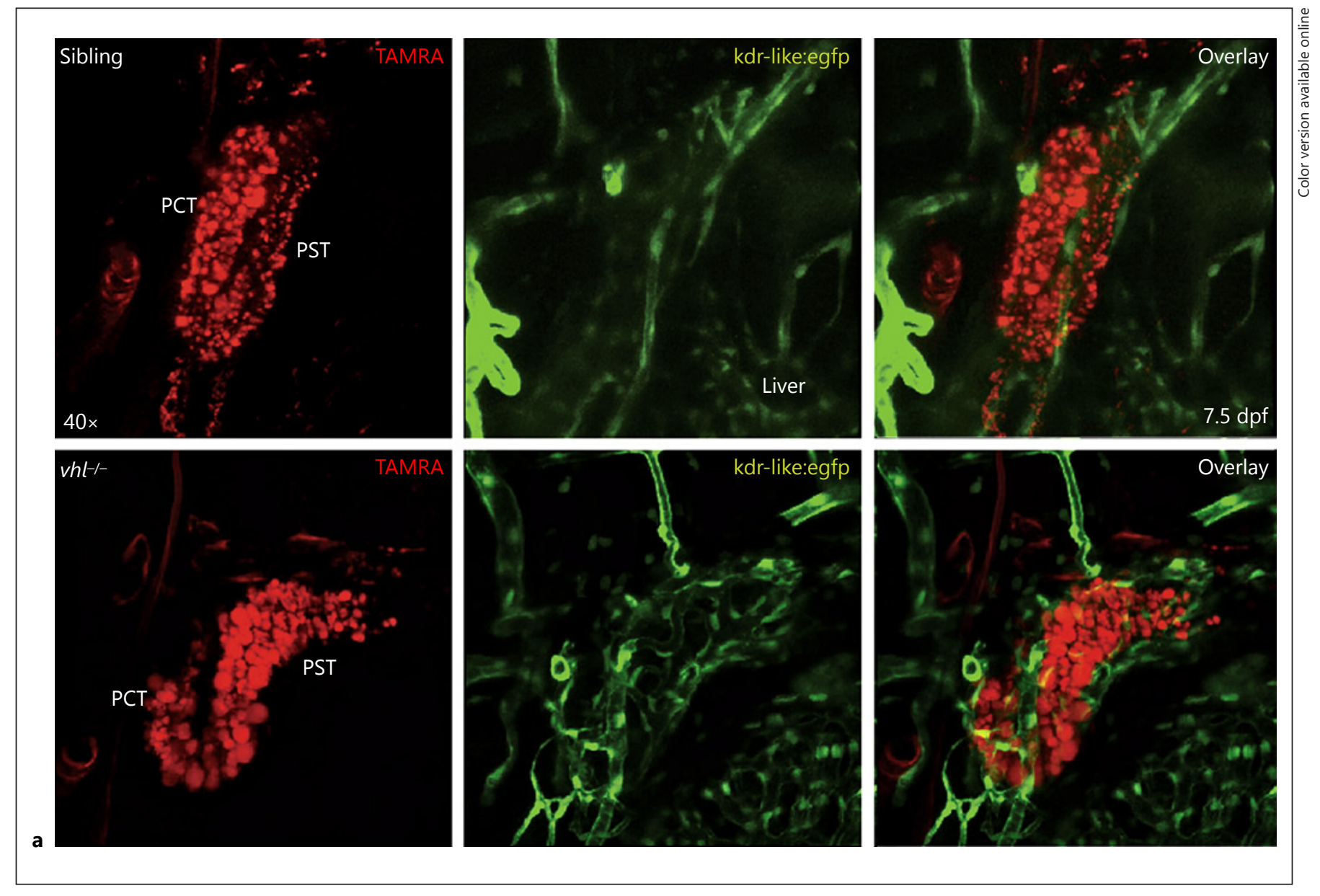

Fig. 4. Neovascularization of the $v h l^{-/-}$proximal tubule does not obviously contribute to the aberrant cell morphology. a Confocal analysis after rhodamine-dextran (TAMRA) injection into $v h l$ mutants and siblings carrying the $k d r$-like:egfp transgene at $7.5 \mathrm{dpf}$. Both mutants and siblings clear TAMRA via the glomerulus (not shown), which is subsequently taken up by endocytosis in the PCT and PST (left panels) or excreted via the cloaca (not shown). While in siblings TAMRA-containing vesicles are small and appear to have a more apical distribution, vesicles in the $v h l^{-/-} \mathrm{PT}$ are larger and appear to fill up most of the PT cell lumen (left panels). Analysis of the blood vessels (middle and right panels) revealed excessive neovascularization of the $v h l^{-/-} \mathrm{PT}$. While in siblings the PT is wrapped around one main blood vessel, in $v h l$ mutants, the PT is surrounded by blood vessels that form a fine cocoon-like structure over the tubular epithelium. Original magnification is $40 \times$. b VEGF receptor inhibitor treatment $(10 \mu \mathrm{M})$ from 2.5 to $5.75 \mathrm{dpf}$ reduced the Vegf-induced $v \mathrm{hl}^{-/-}$neovascularization compared to DMSO $(10 \mu \mathrm{M})$ treated control mutants; however, PT cell morphology was not obviously affected. Left panel, bright field image of the PT. Middle panel, blood vessels around the PT. Right panel, overlay of the bright field image (false-colored red in Photoshop) and blood vessels. Original magnification is $20 \times$. Anterior is to the left and dorsal is up in all images. PCT, proximal convoluted tubule; PST, proximal straight tubule; $\mathrm{PT}$, proximal tubule; hpf, hours post fertilization; dpf, days post fertilization.

(For figure $4 b$ see next page.) erate uptake was observed in these organs (Fig. 5a) with a notable absence of uptake in a distinct distal region of the intestine. Interestingly, in the $\mathrm{vhl}^{-/-}$embryos, dye uptake is observed in this region (Fig. 5b). Vibratome sections of the PT confirm the robust increased uptake and evident vesicle accumulation of the AM1-43 dye in cells (Fig. 5c, d). In support of these data, re-evaluation of microarray RNA expression of $\mathrm{vhl}^{-/-}$zebrafish indi- cates upregulated endocytosis pathway components (online suppl. Fig. 5).

We next asked whether human PT cells lacking VHL exhibit similar changes in vesicle accumulation and trafficking. Using a well-established ccRCC cell line with biallelic VHL mutations (786-0) and isogenic sister clone with reconstituted VHL added back to confirm specificity, we microinjected a fluorescently labelled mCherry- 


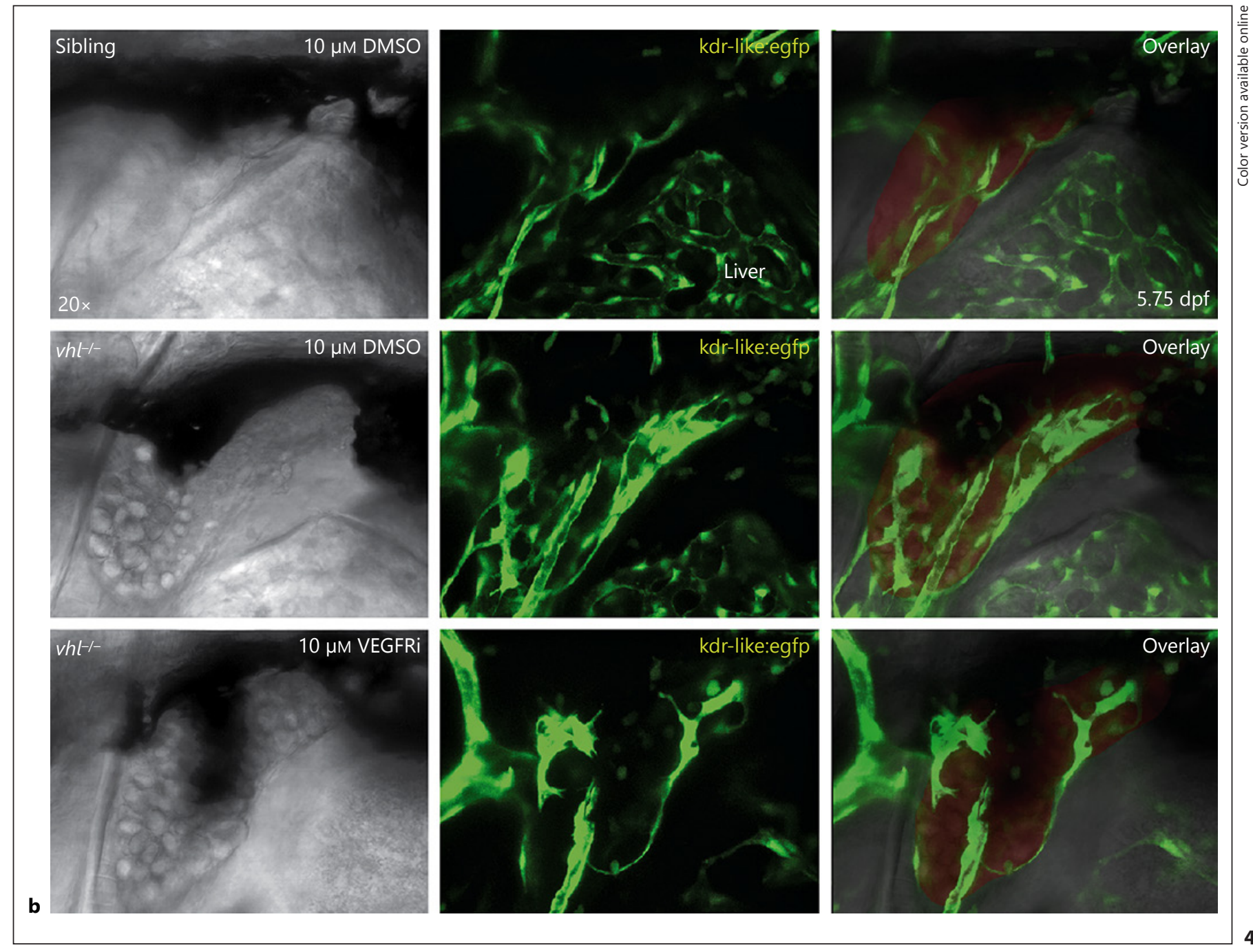

RAB7A construct, as a RAS-related protein involved in endocytosis. After $24 \mathrm{~h}$, we performed live cell imaging of 10 individual fluorescent labelled cells for each condition. In all cells lacking VHL, we observed perinuclear clustering of large RAB7A positive vesicles, which were diffusely spread upon VHL introduction (Fig. 5e, f; online suppl. Movie 1 at https://youtu.be/T3oY3xotQ7s and online suppl. Movie 2 at https://youtu.be/zwesLvf0WlQ). Quantification of the maximum diameter of vesicles reveals that 786-0 cells lacking VHL contain larger vesicles overall when compared to the isogenic reconstituted controls (Fig. 5g). Collectively, these data support a role for VHL in endocytic vesicle uptake and transport.

\section{Discussion}

Here we describe developmental defects of the zebrafish pronephros as a result of the inactivation of the $v h l$ tumour suppressor gene. Notably, the morphological changes of the PT we observe are not secondary to the hypervascularization defect in the fish and may be attributed to endocytic vesicle dynamics. Histological analysis of $\mathrm{Vhl}^{-/-}$cells in the proximal tubule [18] or other tubular segments of the mouse kidney [19] did not reveal a similar defect.

Previously, we have shown that the zebrafish $v h l$ model is characterized by a marked increase in circulating red blood cells [34]. Here, we demonstrate broad glomerular abnormalities due to $v h l$ loss, which are not characterized by altered proliferation, although it remains to be deter- 

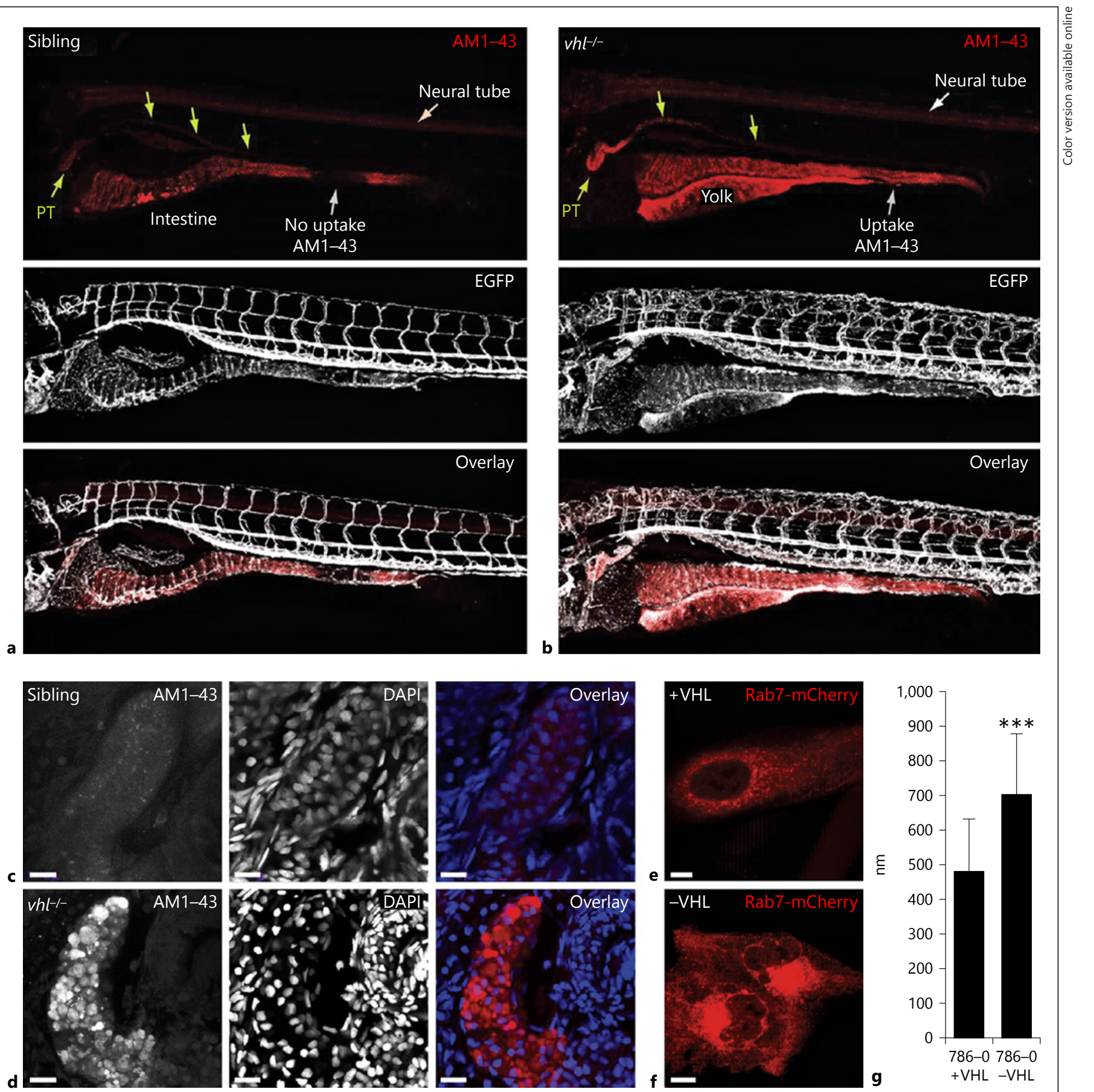

Fig. 5. The endocytic pathway is affected in $v h l$ mutants. a, b Confocal analysis $24 \mathrm{~h}$ after AM1-43 injection is administered into $v \mathrm{hl}$ mutants and siblings carrying the $k d r$-like:egfp transgene at $6 \mathrm{dpf}$. Both mutants and siblings clear AM1-43 via the intestine and PT, with higher fluorescence intensity observed in the mutant embryos. Neural tube staining intensity is equal in both mutant and sibling. $v h l$ mutant embryos retain some of their yolk, which also stains positive for AM1-43. An unstained region was observed in siblings in the more distal region of the intestine, which does stain positive in

the $v h l$ mutant. Anterior is to the left. Original magnification is $10 \times$. c, d Cross sections of the PT of AM1-43 injected embryos shows increased AM1-43 uptake in $v h l^{-/-}$compared to sibling. Scale bar is $10 \mu \mathrm{m}$. e, $\mathbf{f}$ Confocal analysis of micro-injected 786-0 cells shows perinuclear localization of mCherry-RAB7A upon VHL loss. g Quantification of vesicular maximum diameter (size in nanometers). Graphs represent combined results of 2 independent experiments, with 2 times 30 vesicles measured for both conditions. Twotailed $t$ test was used for statistical analysis, showing a $p=6.40658^{-10}$. 
mined whether the glomerular phenotype is primary in nature. Rankin et al. [18] reported that mice with a conditional deletion of $V h l$ in the renal proximal tubule develop glomerular cysts (defined as the glomerular taft occupying $<25 \%$ of the Bowman corpuscle) and proliferative tubular cysts in around $30 \%$ of PEPCK- $\mathrm{Vhl}^{-/-}$mice over 12 months of age. Results, however, are complicated by Cre-transgene expression in the liver, which induced HIF-2a-mediated $E P O$ expression and the development of polycythemia [18]. Transgenic tg6 mice that constitutively overexpress human EPO cDNA develop severe erythrocytoses. Regularly enlarged glomeruli with a widened Bowman space and an enlarged and irregular basement membrane were observed [46]. Monitoring of urine composition in 7-8 month old mice demonstrated significant hematuria and proteinuria indicating that renal ultrafiltration is severely disturbed. Furthermore, high altitude-associated erythrocytoses was found to be positively correlated to the development of proteinuria in humans [47]. These data suggest that the glomerular defects observed in both PEPCK- $\mathrm{Vhl}^{-/}$ mice [18] and $v h l$ mutant zebrafish are likely to be - at least in part - a secondary consequence to the induced polycythemia in both species. Likewise, impairment of podocyte function in zebrafish has been shown to affect glomerular permeability [48]. Loss of $v h l$ in podocytes might contribute to the glomerular defects in $v h l$ mutants, as conditional inactivation of $V h l$ in these cells was shown to severely affect glomerular integrity in mice, leading to hematuria, proteinuria and renal insufficiency from 4 weeks of age in a Cxcr4-dependent and Vegf-independent manner [23]. In another study using the same transgene, mice exhibited a milder phenotype, including glomerulomegaly, an increased Bowman space, glomerulosclerosis and no significant proteinuria [49]. Background-related polymorphisms likely underlie the differences between phenotypes. Interestingly, we show $v h l$ mutants to display glomerulomegaly, dilated Bowman space, dilated cxcr4a-positve capillary loops and podocytes. Although further experimental evidence is warranted, collectively these studies indicate that the $v h l$-induced polycythemia as well as direct loss of $v h l$ in podocytes might lead to defective and leaky ultrafiltration in systemic $v h l$ mutants.

Intriguingly, we find an excessive accumulation of variably sized vesicles of unknown content in the $v h l$ mutant proximal tubule. This becomes apparent by non-invasive visual examination around $3 \mathrm{dpf}$, one day after glomerular filtration in zebrafish embryos starts [42]. Since PT cells become larger over time, this might suggest an accumulative defect, an increased PT reabsorption, or both. Oil Red $\mathrm{O}$ staining indicates that the PT cell accumulates large

Zebrafish VHL Regulates Proximal

Tubule and Glomerulus Integrity amounts of lipids. Hypoxia has previously been shown to instigate a metabolic shift towards glycolysis, potentially leading to lipid accumulation [50]. The accumulation of lipid droplets and/or other vesicle components appears to be independent of the hypervascularization of the pronephros, since reducing vasculature by VEGFR inhibition does not alter the grape-like architecture of the $\mathrm{vhl}^{-{ }^{-}}$pronephros. As the $v h l$ knockout occurs constitutively in all tissues, it makes the spatial and temporal specificities of the mutational effects difficult to discern. Embryonic nephron development requires extensive mesenchymalepithelial interactions. It is therefore not known whether the tubule defect is the result of $v h l$ loss-of-function in the epithelia. We conclude that although the $v \mathrm{hl}^{-/-}$pronephros phenotype may be cell autonomous, multiple cell intrinsic and (physiological) extrinsic factors could contribute. Interestingly, the morphological changes we observe at the cellular level might also be relevant to tumourigenesis associated with VHL loss [4, 59].

Endocytosis and exocytosis are dynamic processes that are key to maintaining osmotic homeostasis in the kidney. Several renal channels and transporters involved in apical and basolateral trafficking utilize a microtubulebased vesicle transport system (reviewed by HammHamm-Alvarez and Sheetz [51]; Rodriguez-Boulan et al. [52]). Interestingly, several studies reported that exposure of rats to microtubule-depolymerizing agents, colchicine and/or nocodazole, led to random cytoplasmic and basolateral distribution of vesicles that normally have an apical localization $[51,53,54]$. pVHL promotes microtubule (MT) stability $[6,55]$ and interacts with MTs through binding with the kinesin- 2 motor protein [56, 57] that is involved in the plus-end directed transport of vesicles and protein cargos along MTs. Although, TAMRA dye excretion tests indicate that endocytosis is altered in $v h l$ mutants, we demonstrate that RAB7 dynamics do appear to be affected by $V H L$ status. We have not examined the role of exocytosis, yet it is intriguing to speculate that our data might reflect a defect in MT-based exocytosis (or transcytosis), involving an impaired kinesin-2 mediated transport towards the basolateral membrane (plus-end). We are currently investigating the exact nature of the $v \mathrm{hl}^{-/-}$proximal tubule and glomerulus abnormalities. Our results indicate that zebrafish $v h l \mathrm{mu}-$ tants mimic key human VHL phenotypes and in combination with different models will likely be required to fully understand this complex disease. 


\section{Acknowledgements}

This project was funded by the Dutch Cancer Association (UU2006-3565), the European Union Seventh Framework Programme under grant agreement number 305608 (EURenOmics) and the Dutch Kidney Foundation (16OI06, and KOUNCIL CP11.18). We gratefully acknowledge Jeroen Korving for tissue sectioning, Kevin van de Ven (Department Pathology, UMC Utrecht) for transmission electron microscopy, the Cell Microscopy Core at the UMC Utrecht for expert support of the confocal, and the animal caregivers at the Hubrecht Institute.

\section{Statement of Ethics}

This study did not require informed consent nor review/approval by the appropriate ethics committee.

\section{Disclosure Statement}

The authors have no conflicts of interest to declare.

\section{References}

1 Nielsen SM, Rhodes L, Blanco I, Chung WK, Eng C, Maher ER, Richard S, Giles RH: Von hippel-lindau disease: genetics and role of genetic counseling in a multiple neoplasia syndrome. J Clin Oncol 2016;34:2172-2181.

2 Van Poppel H, Nilsson S, Algaba F, Bergerheim U, Dal Cin P, Fleming S, Hellsten S, Kirkali Z, Klotz L, Lindblad P, Ljungberg B, Mulders P, Roskams T, Ross RK, Walker C, Wersall P: Precancerous lesions in the kidney. Scand J Urol Nephrol Suppl 2000;205:136-165.

3 Maher ER, Yates JR, Harries R, Benjamin C, Harris R, Moore AT, Ferguson-Smith MA: Clinical features and natural history of von Hippel-Lindau disease. Q J Med 1990;77: 1151-1163.

4 Mandriota SJ, Turner KJ, Davies DR, Murray PG, Morgan NV, Sowter HM, Wykoff CC, Maher ER, Harris AL, Ratcliffe PJ, Maxwell PH: HIF activation identifies early lesions in VHL kidneys: evidence for site-specific tumor suppressor function in the nephron. Cancer Cell 2002;1:459-468.

5 Walther MM, Lubensky IA, Venzon D, Zbar B, Linehan WM: Prevalence of microscopic lesions in grossly normal renal parenchyma from patients with von Hippel-Lindau disease, sporadic renal cell carcinoma and no renal disease: clinical implications. J urol 1995; 154:2010-2014; discussion 2014-2015.

6 Hergovich A, Lisztwan J, Barry R, Ballschmieter P, Krek W: Regulation of microtubule stability by the von Hippel-Lindau tumour suppressor protein pVHL. Nat Cell Biol 2003;5:64-70.

7 Esteban MA, Harten SK, Tran MG, Maxwell $\mathrm{PH}$ : Formation of primary cilia in the renal epithelium is regulated by the von HippelLindau tumor suppressor protein. J Am Soc Nephrol 2006;17:1801-1806.

8 Lutz MS, Burk RD: Primary cilium formation requires von hippel-lindau gene function in renal-derived cells. Cancer Res 2006;66:69036907.

9 Schermer B, Ghenoiu C, Bartram M, Muller RU, Kotsis F, Hohne M, Kuhn W, Rapka M, Nitschke R, Zentgraf H, Fliegauf M, Omran $\mathrm{H}$, Walz G, Benzing T: The von HippelLindau tumor suppressor protein controls ciliogenesis by orienting microtubule growth. J Cell Biol 2006;175:547-554.
10 Lolkema MP, Mans DA, Ulfman LH, Volpi S, Voest EE, Giles RH: Allele-specific regulation of primary cilia function by the von HippelLindau tumor suppressor. Eur J Hum Genet 2008; 16:73-78.

11 Ohh M, Yauch RL, Lonergan KM, Whaley JM, Stemmer-Rachamimov AO, Louis DN, Gavin BJ, Kley N, Kaelin WG Jr, Iliopoulos O: The von Hippel-Lindau tumor suppressor protein is required for proper assembly of an extracellular fibronectin matrix. Molecular cell 1998;1:959-968.

12 Stickle NH, Chung J, Klco JM, Hill RP, Kaelin WG Jr, Ohh M: pVHL modification by NEDD8 is required for fibronectin matrix assembly and suppression of tumor development. Mol Cell Biol 2004;24:3251-3261.

13 Hoffman MA, Ohh M, Yang H, Klco JM, Ivan M, Kaelin WG Jr: von Hippel-Lindau protein mutants linked to type 2C VHL disease preserve the ability to downregulate HIF. Human Mol Genet 2001;10:1019-1027.

14 Haase VH: Hypoxia-inducible factors in the kidney. Am J Physiol Renal Physiol 2006; 291:F271-F281.

15 Ma W, Tessarollo L, Hong SB, Baba M, Southon E, Back TC, Spence S, Lobe CG, Sharma N, Maher GW, Pack S, Vortmeyer AO, Guo C, Zbar B, Schmidt LS: Hepatic vascular tumors, angiectasis in multiple organs, and impaired spermatogenesis in mice with conditional inactivation of the VHL gene. Cancer Res 2003;63:5320-5328.

16 Haase VH, Glickman JN, Socolovsky M, Jaenisch R: Vascular tumors in livers with targeted inactivation of the von Hippel-Lindau tumor suppressor. Proc Natl Acad Sci U S A 2001;98:1583-1588.

17 Kleymenova E, Everitt JI, Pluta L, Portis M, Gnarra JR, Walker CL: Susceptibility to vascular neoplasms but no increased susceptibility to renal carcinogenesis in VHL knockout mice. Carcinogenesis 2004;25:309-315.

18 Rankin EB, Tomaszewski JE, Haase VH: Renal cyst development in mice with conditional inactivation of the von Hippel-Lindau tumor suppressor. Cancer Res 2006;66:2576-2583.
19 Frew IJ, Thoma CR, Georgiev S, Minola A, Hitz M, Montani M, Moch H, Krek W: pVHL and PTEN tumour suppressor proteins cooperatively suppress kidney cyst formation. EMBO J 2008;27:1747-1757.

20 Harlander S, Schonenberger D, Toussaint NC, Prummer M, Catalano A, Brandt L, Moch H, Wild PJ, Frew IJ: Combined mutation in Vhl, Trp53 and Rb1 causes clear cell renal cell carcinoma in mice. Nat Med 2017; 23:869-877.

21 Nargund AM, Pham CG, Dong Y, Wang PI, Osmangeyoglu HU, Xie Y, Aras O, Han S, Oyama T, Takeda S, Ray CE, Dong Z, Berge M, Hakimi AA, Monette S, Lekaye CL, Koutcher JA, Leslie CS, Creighton CJ, Weinhold N, Lee W, Tickoo SK, Wang Z, Cheng EH, Hsieh JJ: The SWI/SNF protein PBRM1 restrains VHL-loss-driven clear cell renal cell carcinoma. Cell Rep 2017;18:2893-2906.

22 Bailey ST, Smith AM, Kardos J, Wobker SE, Wilson HL, Krishnan B, Saito R, Lee HJ, Zhang J, Eaton SC, Williams LA, Manocha U, Peters DJ, Pan X, Carroll TJ, Felsher DW, Walter V, Zhang Q, Parker JS, Yeh JJ, Moffitt RA, Leung JY, Kim WY: MYC activation cooperates with Vhl and Ink4a/Arf loss to induce clear cell renal cell carcinoma. Nat Commun 2017;8:15770

23 Ding M, Cui S, Li C, Jothy S, Haase V, Steer BM, Marsden PA, Pippin J, Shankland S, Rastaldi MP, Cohen CD, Kretzler M, Quaggin SE: Loss of the tumor suppressor Vhlh leads to upregulation of Cxcr4 and rapidly progressive glomerulonephritis in mice. Nat Med 2006;12:1081-1087.

24 Thoma CR, Frew IJ, Hoerner CR, Montani M, Moch H, Krek W: pVHL and GSK3beta are components of a primary cilium-maintenance signalling network. Nat Cell Biol 2007; 9:588-595.

25 Guinot A, Lehmann H, Wild PJ, Frew IJ: Combined deletion of Vhl, Trp53 and Kif3a causes cystic and neoplastic renal lesions. J Pathol 2016;239:365-373.

26 Drummond IA: Kidney development and disease in the zebrafish. J Am Soc Nephrol 2005; 16:299-304. 
27 Wingert RA, Davidson AJ: The zebrafish pronephros: a model to study nephron segmentation. Kidney Int 2008;73:1120-1127.

28 van de Hoek G, Nicolaou N, Giles RH, Knoers NV, Renkema KY, Bongers EM: Functional models for congenital anomalies of the kidney and urinary tract. Nephron 2015;129: 62-67.

29 Noonan HR, Metelo AM, Kamei CN, Peterson RT, Drummond IA, Iliopoulos O: Loss of VHL in the zebrafish pronephros recapitulates early stages of human clear cell renal cell carcinoma. Dis Model Mech 2016;9:873-884

30 Wienholds E, van Eeden F, Kosters M, Mudde J, Plasterk RH, Cuppen E: Efficient target-selected mutagenesis in zebrafish. Genome Res 2003;13:2700-2707.

31 Jin SW, Beis D, Mitchell T, Chen JN, Stainier DY: Cellular and molecular analyses of vascular tube and lumen formation in zebrafish. Development 2005;132:5199-5209.

32 Thisse C, Thisse B, Schilling TF, Postlethwait $\mathrm{JH}$ : Structure of the zebrafish snaill gene and its expression in wild-type, spadetail and no tail mutant embryos. Development 1993;119: 1203-1215.

33 Chong SW, Emelyanov A, Gong Z, Korzh V: Expression pattern of two zebrafish genes, $\mathrm{cx}$ cr4a and cxcr4b. Mech Dev 2001;109:347354.

34 van Rooijen E, Voest EE, Logister I, Korving J, Schwerte T, Schulte-Merker S, Giles RH, van Eeden FJ: Zebrafish mutants in the von Hippel-Lindau tumor suppressor display a hypoxic response and recapitulate key aspects of Chuvash polycythemia. Blood 2009;113: 6449-6460.

35 Schulte-Merker S: Looking at embryos; in Nusslein-Volhard C, Dahm R (eds): Zebrafish, a Practical Approach. Oxford, Oxford University Press, 2002.

36 Westerfield M: The Zebrafish Book. A Guide for the Laboratory Use of Zebrafish (Danio rerio), ed 3rd Edition. Eugene, University of Oregon Press, 1995.

37 Paffett-Lugassy NN, Zon LI: Analysis of hematopoietic development in the zebrafish. Methods Mol Med 2005;105:171-198.

38 Mans DA, Vermaat JS, Weijts BG, van Rooijen E, van Reeuwijk J, Boldt K, Daenen LG, van der Groep P, Rowland BD, Jans JJ, Roepman R, Voest EE, van Diest PJ, Verhaar MC, de Bruin A, Giles RH: Regulation of E2F1 by the von Hippel-Lindau tumour suppressor protein predicts survival in renal cell cancer patients. J Pathol 2013;231:117129.
39 Wingert RA, Selleck R, Yu J, Song HD, Chen Z, Song A, Zhou Y, Thisse B, Thisse C, McMahon AP, Davidson AJ: The cdx genes and retinoic acid control the positioning and segmentation of the zebrafish pronephros. PLoS Genet 2007;3:1922-1938.

40 Page T, Hodgkinson AD, Ollerenshaw M, Hammonds JC, Demaine AG: Glucose transporter polymorphisms are associated with clear-cell renal carcinoma. Cancer genetics and cytogenetics 2005;163:151-155.

41 van Rooijen E, Voest EE, Logister I, Bussmann J, Korving J, van Eeden FJ, Giles RH, Schulte-Merker S: von Hippel-Lindau tumor suppressor mutants faithfully model pathological hypoxia-driven angiogenesis and vascular retinopathies in zebrafish. Dis Model Mech 2010;3:343-353.

42 Drummond IA, Majumdar A, Hentschel H, Elger M, Solnica-Krezel L, Schier AF, Neuhauss SC, Stemple DL, Zwartkruis F, Rangini Z, Driever W, Fishman MC: Early development of the zebrafish pronephros and analysis of mutations affecting pronephric function. Development 1998;125:4655-4667.

43 Zhao C, Malicki J: Genetic defects of pronephric cilia in zebrafish. Mech Dev 2007;124: 605-616.

44 Kramer-Zucker AG, Olale F, Haycraft CJ, Yoder BK, Schier AF, Drummond IA: Cilia-driven fluid flow in the zebrafish pronephros, brain and Kupffer's vesicle is required for normal organogenesis. Development 2005;132: 1907-1921.

45 Betz WJ, Mao F, Bewick GS: Activity-dependent fluorescent staining and destaining of living vertebrate motor nerve terminals. J Neurosci 1992;12:363-375.

46 Heinicke K, Baum O, Ogunshola OO, Vogel J, Stallmach T, Wolfer DP, Keller S, Weber K, Wagner PD, Gassmann M, Djonov V: Excessive erythrocytosis in adult mice overexpressing erythropoietin leads to hepatic, renal, neuronal, and muscular degeneration. Am J Physiol 2006;291:R947-R956.

47 Jefferson JA, Escudero E, Hurtado ME, Kelly JP, Swenson ER, Wener MH, Burnier M, Maillard M, Schreiner GF, Schoene RB, Hurtado A, Johnson RJ: Hyperuricemia, hypertension, and proteinuria associated with high-altitude polycythemia. Am J Kidney Dis 2002;39:1135-1142.
48 Kramer-Zucker AG, Wiessner S, Jensen AM, Drummond IA: Organization of the pronephric filtration apparatus in zebrafish requires Nephrin, Podocin and the FERM domain protein Mosaic eyes. Dev Biol 2005;285: 316-329.

49 Brukamp K, Jim B, Moeller MJ, Haase VH: Hypoxia and podocyte-specific Vhlh deletion confer risk of glomerular disease. Am J Physiol Renal Physiol 2007;293:F1397F1407.

50 Huang D, Li T, Li X, Zhang L, Sun L, He X, Zhong X, Jia D, Song L, Semenza GL, Gao P, Zhang H: HIF-1-mediated suppression of acyl-CoA dehydrogenases and fatty acid oxidation is critical for cancer progression. Cell Rep 2014;8:1930-1942.

51 Hamm-Alvarez SF, Sheetz MP: Microtubuledependent vesicle transport: modulation of channel and transporter activity in liver and kidney. Physiol Rev 1998;78:1109-1129.

52 Rodriguez-Boulan E, Kreitzer G, Musch A: Organization of vesicular trafficking in epithelia. Nat Rev Mol Cell Biol 2005;6:233-247.

53 Elkjaer ML, Birn H, Agre P, Christensen EI, Nielsen S: Effects of microtubule disruption on endocytosis, membrane recycling and polarized distribution of Aquaporin-1 and gp330 in proximal tubule cells. Eur J Cell Biol 1995;67:57-72.

54 Gutmann EJ, Niles JL, McCluskey RT, Brown D: Colchicine-induced redistribution of an apical membrane glycoprotein (gp330) in proximal tubules. Am J Physiol 1989;257:C397-C407.

55 Lolkema MP, Mehra N, Jorna AS, van Beest M, Giles RH, Voest EE: The von HippelLindau tumor suppressor protein influences microtubule dynamics at the cell periphery. Exp Cell Res 2004;301:139-146.

56 Lolkema MP, Mans DA, Snijckers CM, van Noort M, van Beest M, Voest EE, Giles RH: The von Hippel-Lindau tumour suppressor interacts with microtubules through kinesin-2. FEBS Lett 2007;581:4571-4576.

57 Mans DA, Lolkema MP, van Beest M, Daenen LG, Voest EE, Giles RH: Mobility of the von Hippel-Lindau tumour suppressor protein is regulated by kinesin-2. Exp Cell Res 2008;314: 1229-1236.

$58 \mathrm{Hu}$, Flockhart I, Vinayagam A, Bergwitz C, Berger B, Perrimon N, Mohr SE: An integrative approach to ortholog prediction for disease-focused and other functional studies. BMC Bioinformatics 2011;12:357.

59 Krishnan B, Truong LD: Renal epithelial neoplasms: the diagnostic implications of electron microscopic study in 55 cases. Hum Pathol 2002;33:68-79. 